Rev. Inst. Flor. v. 30 n. 1 p. 47-51 jun. 2018

http://dx.doi.org/10.24278/2178-5031.201830104

ISSN impresso 0103-2674/on-line 2178-5031

\title{
INFLUÊNCIA DA ALTURA E DIÂMETRO DAS CEPAS NA REBROTA DE Eucalyptus SPP ${ }^{1}$
}

\author{
INFLUENCE OF HEIGHT AND DIAMETER OF STUMPS IN THE Eucalyptus SPP \\ REGROWTH
}

\author{
Danusia Silva LUZ2,3; Luis Carlos de FREITAS²; Caio Jander Nogueira PRATES²; \\ Ana Paula da Silva BARROS ${ }^{2}$
}

\begin{abstract}
RESUMO - Muitas espécies de Eucalyptus apresentam alta capacidade de regeneração das cepas após o abate da árvore, o que permite conduzir o povoamento em mais de uma rotação. Sendo assim, o objetivo deste trabalho foi avaliar a influência da altura de corte e diâmetro das cepas no vigor das brotações de Eucalyptus spp. O estudo foi realizado em um povoamento de Eucalyptus spp, com espaçamento de 2,5 X 2,5 metros. A área em estudo pertence a uma empresa de base florestal, situada no município de Barra do Choça-BA. As alturas das cepas foram medidas com auxílio de uma régua graduada, sendo a altura dos brotos, mensurada por meio de uma trena. Os diâmetros das cepas e brotos foram quantificados por meio de uma suta. Os dados obtidos foram rodados no programa estatístico SAEG versão 9.1, sendo os parâmetros avaliados pela correlação de Spearman. A altura da cepa mostrou baixa correlação com o quantitativo de brotação. O diâmetro da cepa não mostrou correlação com o número de brotos. Não houve correlação significativa para o diâmetro da cepa e a altura do maior broto. Não houve correlação entre diâmetro da cepa e diâmetro dos brotos. Apesar da importância no planejamento silvicultural, principalmente para pequenos e médios produtores florestais, observa-se uma escassez de trabalhos mais recentes na área em questão.
\end{abstract}

Palavras-chave: Regeneração; vigor; produtividade.

\begin{abstract}
Many species of Eucalyptus present a high capacity of regeneration of the stumps after the tree has been cut and felled, which allows to lead the population in more than one rotation. Therefore, the aim of this work was to evaluate the influence of height and diameter of the strains on the vigor of Eucalyptus spp. The study was carried out in a plantation of Eucalyptus spp, with the spacing of 2.5 X 2.5 meters. The study area belongs to a forest-based company located in the town of Barra do Choça-BA. The heights of the strains were measured by using a graduated ruler; the height of the shoots was measured with a tape measure. The diameters of the strains and shoots were quantified through a sliding $\mathrm{T}$ bevel. The data obtained were run in the statistical program SAEG version 9.1, and the parameters were evaluated by the Spearman correlation. The height of the strain showed a low correlation with the sprouting quantity. The diameter of the stump showed no correlation with the number of shoots. There was no significant correlation for the diameter of the stump and height of the largest shoot. There was no correlation between strain diameter and shoot diameter. Despite the importance of silvicultural planning, especially for small and medium forest producers, we could observe a shortage of more recent work in this area.
\end{abstract}

Keywords: Regeneration; Vigor; Productivity.

$\overline{1}$ Recebido para análise em 25.02.2018. Aceito para publicação em 14.05.2018.

${ }^{2}$ Universidade Estadual do Sudoeste da Bahia (UESB), Campus Vitória da Conquista, Estrada do Bem Querer, Km 04, CEP: 45083-900, Vitória da Conquista, BA, Brasil.

${ }^{3}$ Autor para correspondência: Danusia Silva Luz - danflorestal@hotmail.com 


\section{INTRODUÇÃO}

As florestas plantadas, além do fornecimento de matéria-prima para suprimento das indústrias de base florestal, apresentam um papel ecológico e ambiental relevante por amenizar a pressão sobre as florestas nativas. Dentro deste contexto, o eucalipto tem apresentado destaque devido às características inerentes à espécie, destacando-se a sua vantagem na boa capacidade de rebrota (Higa e Sturion, 1991) e adaptações às condições edofoclimáticas (Lima, 1996).

A condução de eucalipto pelo regime talhadia tem sido uma prática consolidada para atender a demanda de madeira nos mais variados segmentos florestais. De acordo com Camargo et al. (1997), este método tem a vantagem de dispensar algumas das atividades de implantação florestal, como preparo do solo e produção de mudas, o que consequentemente levaria a uma redução de custos para o empreendimento.

Todavia, a sobrevivência e produtividade dos indivíduos conduzidos pelo regime talhadia dependem de diversos fatores inerentes ao local e à espécie estudada, tais como regime hídrico (Gonçalves et al., 2014), nutrientes do solo (Simões e Couto, 1985), presença de gemas adventícias e lignotuber (Wildy e Pate, 2002; Walters et al., 2005), além da altura de corte das cepas, fator este que reflete na capacidade de rebrota, sobretudo, nas espécies que apresentam uma baixa capacidade de emitir brotação. Segundo Ferrari et al. (2005), a altura do corte pode apresentar um efeito positivo em relação à sobrevivência das cepas, uma vez que tocos mais altos condicionam um maior número de gemas ativas, o que aumenta a possibilidade de brotação. Outro fator de grande relevância no vigor das brotações é o diâmetro das cepas (Graça e Toth, 1990). Árvores de maiores diâmetros apresentam sistemas radiculares mais desenvolvidos, aumentando a capacidade de absorção em água e nutrientes no solo (Oliveira et al., 2008), levando, consequentemente, à formação de brotações mais vigorosas, ou seja, de maior altura e diâmetro.

Dada as grandes vantagens na condução de florestas pelo regime de talhadia, seja pelo maior taxa de crescimento inicial das brotações comparadas com as mudas (Reis e Reis, 1997); redução de custos nos processos de implantação (Camargo et al., 1997) ou facilidade de planejamento da produção madeireira a curto e médio prazos (Stape, 1997), objetivou-se com esta pesquisa avaliar a influência da altura de corte e do diâmetro das cepas no vigor de brotações de Eucalyptus spp.

\section{MATERIAL E MÉTODOS}

O estudo foi realizado em um povoamento de Eucalyptus spp, com espaçamento de 2,5 X 2,5 metros. $\mathrm{O}$ empreendimento pertence a uma empresa de base florestal, situada no município de Barra do Choça-BA, localizado sob as coordenadas geográficas $14^{\circ} 52^{\prime} \mathrm{S}$ e $40^{\circ} 39 \mathrm{O}$, com altitude média de $900 \mathrm{~m}$. O local possui clima Tropical Sub-úmido, representando uma área de transição entre o clima úmido, localizado a leste, e o clima semi-árido, localizado a oeste, (Prefeitura Municipal de Barra do Choça, 2010).

Os dados foram coletados 90 dias após o corte, sendo a floresta de primeira rotação, com aproximadamente sete anos de idade. As alturas das cepas foram medidas com auxílio de uma régua graduada, sendo a altura dos brotos mensurada por meio de uma trena. Para as cepas que apresentaram o corte em bisel foram tomadas duas alturas para obtenção da média, uma no lado mais alto e a outra no lado mais baixo. Os diâmetros das cepas e brotos foram quantificados por meio de uma suta. Realizou-se um caminhamento em linhas sequenciais dentro do povoamento onde foram avaliados um total de 25 plantas.

Os dados obtidos foram rodados no programa estatístico SAEG versão 9.1, sendo realizada uma análise de correlação linear de Spearman para verificação da relação entre as seguintes variáveis: altura e diâmetro das cepas, número de brotos, altura do maior broto e média do diâmetro dos brotos.

Após análise estatística, projetou-se um ajuste de modelos para as variáveis supracitadas, utilizando planilhas eletrônicas do Excel.

\section{RESULTADOS E DISCUSSÃO}

A espécie apresentou boa capacidade de rebrota na região estudada, porém, verificando a correlação de Spearman, observou-se que a maioria das variáveis não se apresentaram correlacionadas positivamente (Tabela 1). 
LUZ, D.S. et al. Rebrota em cepas de Eucalyptus spp.

Tabela 1. Coeficientes de correlação de Spearman entre as variáveis: diâmetro do toco (DIAT), altura do toco (ALTT), número de brotos (NBR), média do diâmetro dos brotos (MEDB) e altura do maior broto (ALTMB).

Table 1. Spearman correlation coefficients among the variables: stump diameter (DIAT), stump height (ALT), number of shoots (NBR), mean shoot diameter (DBH) and height of largest shoot (ALTMB).

\begin{tabular}{cccccc}
\hline & DIAT & ALTT & NBR & MEDB & ALTMB \\
\hline DIAT & 1 & & & & \\
ALTT & $-0,2424^{*}$ & 1 & & & \\
NBR & $-0,0554^{\mathrm{ns}}$ & $0,2658^{*}$ & 1 & & \\
MEDB & $-0,2800^{*}$ & $-0,1384 *$ & $-0,4341^{*}$ & 1 & \\
ALTMB & 0,0194 ns & $0,1804 *$ & $0,4417^{*}$ & $-0,0754$ ns & 1,000 \\
\hline
\end{tabular}

*Significativo em $5 \%$ de probabilidade de erro, pelo teste " $\mathrm{t}$ ". *Significant at $5 \%$ probability of error, by "t" test. ns Não significativo. ${ }^{\mathrm{ns}}$ Not significant.

Cepas de maiores alturas apresentaram maior número de brotações (Figura 1), com maior probabilidade de destaque em relação à altura do maior broto (Figura 2). Quanto maior a altura de corte maior é a possibilidade de sobrevivência da touça (Ferrari et al., 2005). Cepas maiores contemplam uma maior quantidade de gemas de brotação (Souza et al., 1991), favorecendo uma correlação positiva com o número de brotos emitidos. Na pesquisa em questão, observou-se, contudo, uma correlação fracamente relacionada $(0,2658)$, conforme observado na tabela 1 .

O maior número de brotação na fase inicial aumenta também o efeito da competição dos brotos, proporcionando um impacto negativo em relação à média do diâmetro dos brotos (Figura 3). Cada broto deixado na cepa comporta-se semelhantemente a uma planta isolada e contribui para aumentar a pressão sobre os recursos do meio (Reis e Reis, 1997).

O diâmetro das cepas não mostrou correlação positiva com o número de brotações (Tabela 1), o que implica dizer que a área física de casca proporcionada pelo maior diâmetro das cepas não influenciou no aparecimento de novas gemas de brotação. Simões et al. (1972), avaliando o comportamento da brotação de Eucalyptus saligna, concluíram que o aumento do diâmetro das árvores não influiu na capacidade de brotação das cepas. Este aspecto foi observado por Pereira et al. (1980), os quais verificaram aumento do vigor das cepas (relacionado ao crescimento em altura), nas classes de 2,5 $\mathrm{cm}$ a $36,6 \mathrm{~cm}$. Estes autores constataram um decréscimo na altura dos brotos a partir de 36,6 cm de diâmetro. Todavia, para o presente estudo, não se observou correlação significativa em relação ao diâmetro das cepas com altura do maior broto (Tabela 1; Figura 4).

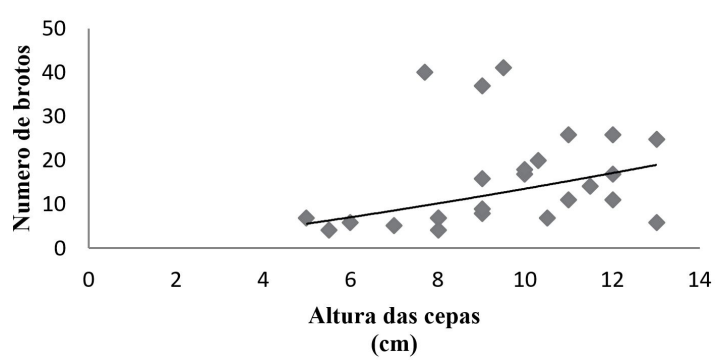

Figura 1. Dispersão do número de brotos e altura do maior broto em relação à altura das cepas.

Figure 1. Dispersion of the number of shoots and height of the largest shoot in relation to the height of the strains.

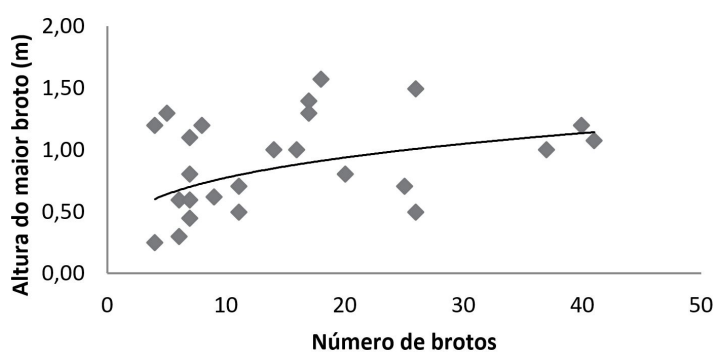

Figura 2. Altura do maior broto em relação ao número de brotações.

Figure 2. Height of the largest shoot in relation to the number of shoots.

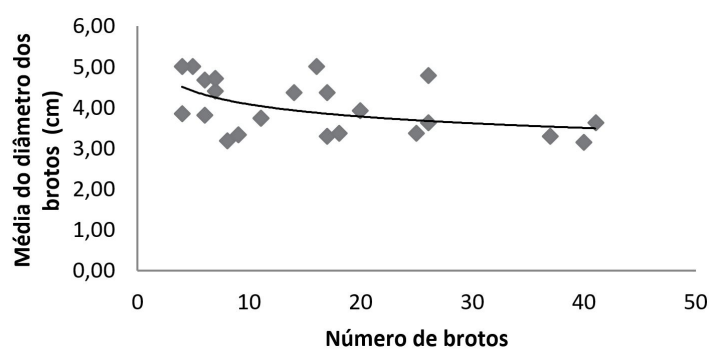

Figura 3. Média do diâmetro dos brotos em relação ao quantitativo de brotação.

Figure 3. Average sprout diameter in relation to sprout quantitative. 


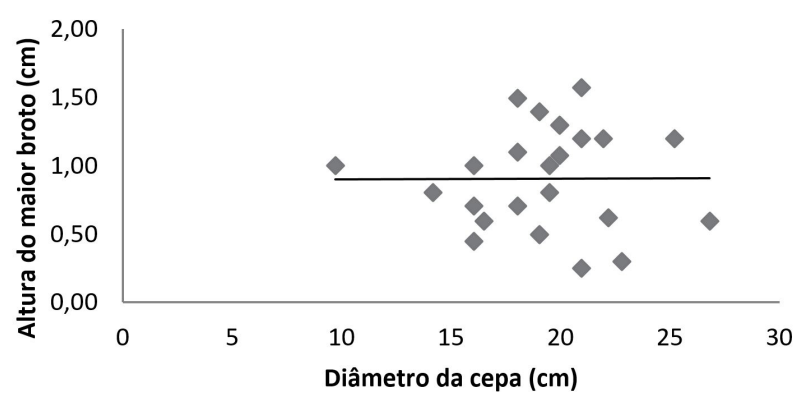

Figura 4. Distribuição da altura do maior broto em relação ao diâmetro das cepas.

Figure 4. Distribution of the number of shoots (A) and height of the largest shoot $(\mathrm{B})$ in relation to the diameter of the strains.

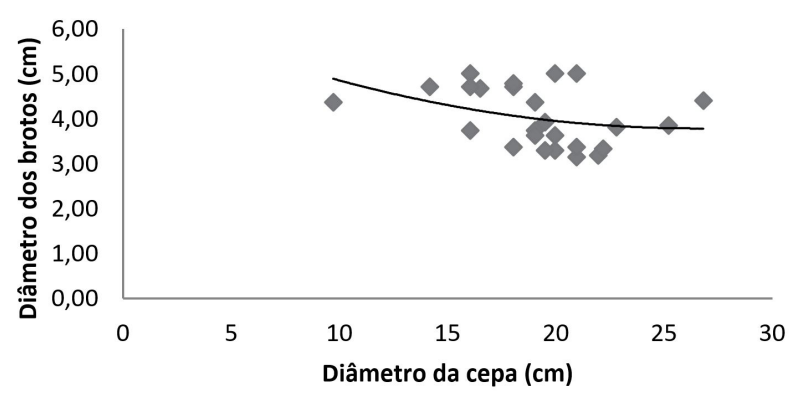

Figura 5. Diâmetro dos brotos em relação ao diâmetro da cepa.

Figure 5. Diameter of the shoots in relation to the diameter of the strain.

Percebeu-se uma correlação negativa entre o diâmetro do toco e a média do diâmetro dos brotos (Tabela 1; Figura 5). Segundo estudos da Ferrari et al. (2005), o crescimento em diâmetro mostra-se menos expressivo na fase inicial quando comparado ao desenvolvimento em altura, tal aspecto pode justificar em parte a correlação apresentada.

\section{CONCLUSÃO}

Diante do presente estudo, recomenda-se avaliar o vigor das brotações em intervalos de tempos sucessivos até que se interrompa a emissão de novos brotos. Por retratar um estudo de caso, resultantes divergentes podem ocorrer em virtude da variação climática, edáfica, período de rotação avaliado (primeiro, segundo ou terceiro cortes), idade dos povoamentos, espaçamento adotado, dentre outros aspectos.
Apesar de grande importância no planejamento silvicultural, principalmente para pequenos e médios produtores florestais, observa-se uma escassez de trabalhos mais recentes na área em questão.

\section{AGRADECIMENTOS}

À Universidade Estadual do Sudoeste da Bahia (UESB), pela concessão da bolsa de incentivo à pesquisa.

\section{REFERÊNCIAS BIBLIOGRÁFICAS}

CAMARGO, F.R.A.; SILVA, C.R.; STAPE, J.L. Resultados experimentais da fase de emissão de brotação em Eucalyptus manejado por talhadia. Série Técnica - IPEF, v. 11, n. 30, p. 115-122, 1997.

FERRARI, M.P.; FERREIRA, C.A.; SILVA, H.D. Condução de plantios de Eucalyptus em sistema de talhadia. Colombo: Embrapa Florestas, 2005. p. 28. (Documentos, n. 104).

GONÇALVES, J.L.M. et al. Produtividade de plantações de eucalipto manejadas nos sistemas de alto fuste e talhadia, em função de fatores edafoclimáticos. Revista Scientia Forestalis, Piracicaba, v. 42, n. 103, p. 411-419, 2014

GRAÇA, M.E.C.; TOTH, V.B.R. Rebrota de Eucalyptus dunni: a influência da altura, diâmetro e procedência no vigor das brotações. Boletim de Pesquisa Florestal, Colombo, n. 20, p. 49-57, 1990.

HIGA, R. C. V.; STURION, J. A. Avaliação da brotação de treze espécies de Eucalyptus na região de Uberaba - MG. Boletim de Pesquisa Florestal, Colombo, n. 22/23, p.79-86, 1991.

LIMA, D.G. Desenvolvimento e aplicação de um modelo de suporte à decisão sobre multiprodutos de povoamentos de eucalipto. 1996. 80 f. Dissertação (Mestrado em Ciência Florestal) - Universidade Federal de Viçosa.

OLIVEIRA, C.H.R. et al. Área foliar e biomassa de plantas intactas e de brotações de plantas jovens de clone de eucalipto em sistemas agrossilvipastoris. Revista Árvore, v. 32, n. 1, p. 59-68, 2008. http:// dx.doi.org/10.1590/S0100-67622008000100008. 
PEREIRA, A.R. et al. Efeito do diâmetro das cepas no desenvolvimento de brotações de Eucalyptus spp. Revista Árvore, v. 4, n. 2, p. 215-220, 1980.

PREFEITURA MUNICIPAL DE BARRA DO CHOÇA. Plano Municipal de Educação. Barra do Choça: Prefeitura Municipal de Barra do Choça, 2010.

REIS, G.G.; REIS, M.G.F. Fisiologia da brotação de eucalipto com ênfase nas suas relações hídricas. Série Técnica IPEF, v. 11, n. 30, p. 9-22, 1997.

SIMÕES, J.L.; COUTO, N.A.S. Efeito do numero de brotos e da fertilização mineral sobre o crescimento da brotação de Eucalyptus Saligna Smith, em segunda rotação. IPEF, v. 31, p. 23-32, 1985.

SIMÕES, J.W. et al. Efeito da ferramenta de corte sobre a regeneração do eucalipto. IPEF, n. 4, p. 3-10, 1972.
SOUZA, A.J. et al. Observações preliminares de alguns fatores que afetam a brotação do eucalipto. Piracicaba: IPEF, 1991. 6 p. (Circular técnica, n. 177).

STAPE, J.L. Planejamento global e normatização de procedimentos operacionais da talhadia simples em Eucalyptus. IPEF, v. 11, n. 30, p. 51-62, 1997.

WALTERS, J.R.; BELL, T.L.; READ, S. Intra-specific variation in carbohydrate reserves and sprouting ability in Eucalyptus obliqua ssedlings. Australian Journal of Botany, v. 53, n. 3, p. 195-203, 2005. http://dx.doi.org/10.1071/BT04016.

WILDY, D.T.; PATE, J.S. Quantifyng above - and below - ground growth responses of the western Australian oil mallee, Eucalyptus kochii subsp. Plenissima, to contrasting decapitation regimes. Annals of Botany, v. 90, n. 2, p. 185-197, 2002. http://dx.doi.org/10.1093/ aob/mcf166. PMid:12197516. 